\title{
LA CONSTRUCCIÓN DEL CONOCIMIENTO PROYECTUAL EN EL DISEÑO ARQUITECTÓNICO: DIMENSIONES COGNOSCITIUAS Y EPISTÉMICAS IMPLICADAS EN EL PROCESO EDUCATIUO
}

\author{
Carlos E., BURGOS \\ carloseb@arq.unne.edu.ar / carloseduardo.burgos@gmail.com \\ Director del Instituto Interdisciplinario de Investigación en Diseño FAU-UNNE. Profesor titular ordinario de Teoría del Diseño I y II, carrera de \\ Arquitectura, FAU-UNNE.
}

Palabras Clave: Conocimiento proyectual; cognición; método.

Keywords: proyectual knowledge; cognition; method

\section{RESUMEN}

El proyecto arquitectónico es considerado aquí como un proceso cognitivo y una praxis generadora de conocimientos, representando un replanteo sobre la visión clásica. Se abordan diferentes tipos de conocimientos proyectuales, tanto los explícito-conceptuales como los implícito-procedimentales. La metodología triangula un análisis teórico y un diseño descriptivo-observacional para estudiar los protocolos del proyecto y sus abordajes curriculares. Los datos evidencian operaciones cognitivas y representacionales complejas, aunque no sean consideradas como objetos de estudio en los modelos educativos clásicos. Aporto un modelo praxeológico de variables para comprender el funcionamiento del proyecto y algunos lineamientos para producir cambios en las actividades de formación.

\begin{abstract}
The architectural project is considered here as a cognitive process and a generating praxis of knowledge, representing a stakeout on the classical view. Different types of knowledge are addressed here, the explicit-conceptual and the implicit procedural. The methodology triangulates a theoretical analysis and a descriptive-observational design to study the protocol of the project and its curricular approaches. The data show cognitive operations and complex representational, although they are not considered as objects of study in classical educational models. I provided a praxeological model of variables to understand the functioning of the project and some guidelines to produce changes in formation activities.
\end{abstract}




\section{INTRODUCCIÓN}

Existe un marcado consenso en afirmar que el "taller de arquitectura" es el espacio privilegiado de síntesis de la carrera y el lugar donde se integra la multiplicidad de saberes que intervienen en los diferentes procesos de formación. Es allí donde los futuros profesionales ponen en práctica sus capacidades conceptuales y operacionales para el proyecto. Sin embargo, persiste una cuestión problemática poco abordada en la actualidad: ¿cuáles son los conocimientos genuinamente proyectuales implicados en el proceso de diseño y qué tipo de estrategias educativas los promueven?

Creo - a modo de hipótesis - que existe un alto grado de indeterminación sobre cuál es el tipo de conocimiento proyectante que el alumno efectivamente construye en su práctica proyectual, y de incertidumbre sobre cuáles son los objetos de estudio que merecen ser focalizados por estrategias didácticas adecuadas para conducir modelos de gestión pedagógica coherentes con este espacio curricular. Esto significa que los saberes y competencias centrales en la ejecución de las tareas más relevantes de un arquitecto quedan sumidos en una cierta oscuridad desde el punto de vista epistémico, y en una preocupante incertidumbre educativa.

El propósito fundamental de este trabajo es aportar elementos para elucidar la naturaleza del conocimiento proyectual implicado en la labor de proyecto, identificando la acción del diseñador como una praxis proyectante a la vez conceptualestructurante y procedimental-metodológica'. Como consecuencia de esta intención, pretendo de manera específica (i) proponer un modelo plausible sobre la constelación de variables asociadas a los

1 En el marco de este trabajo diseño y proyecto serán utilizados como sinónimos. Reconozco que es posible realizar un contraste entre ambos, aunque no es el propósito de esta investigación ponerlo de relieve. conocimientos proyectuales; (ii) señalar posibles objetos de estudio para orientar estrategias de formación orientadas a su construcción y (iii) esbozar una crítica sobre el modelo educativo dominante en las disciplinas proyectuales.

Como hipótesis auxiliar sostendré que aún persisten modelos o paradigmas educativos tradicionales, derivados de la concepción que se sostiene en el proyecto que centra más las acciones educativas en el perfeccionamiento de un "hacer" que en una praxis cognitivo-tecnológica compleja con impacto en la configuración de la cultura actual. Desde este punto de vista, se cuestiona la concepción del proyecto como un oficio o una mera actividad profesional, entendiéndose esto último como un conjunto de conocimientos, prácticas y experticias ligadas a situaciones problemáticas más o menos conocidas y rutinarias. El proyecto es un proceso cognitivo complejo, de reconstrucción simbólica y de transformación de la realidad a partir de los nuevos niveles de significación que portan sus objetos proyectuales al reconfigurar las tramas de la cultura. Alterar las bases epistémicas de su conceptualización implica, también, modificar las líneas de investigación asociadas con él y las modalidades de formación tanto en el grado como en el posgrado. Sin embargo, esta posibilidad no será factible si antes no presentamos un modelo plausible de los conocimientos que el diseñador va constituyendo en los mecanismos de su praxis proyectual.

Para el abordaje de esta situación problemática, propongo el diseño de una metodología mixta para triangular los datos de análisis sobre el objeto de estudio en esta investigación:

(i) un enfoque teórico-epistémico de sistematización conceptual, que utiliza la crítica analítica sobre el estado de la cuestión, presente en las principales contribuciones publicadas sobre el campo; 
“...los saberes y competencias centrales en la ejecución de las tareas más relevantes de un arquitecto quedan sumidos en una cierta oscuridad desde el punto de vista epistémico, y en una preocupante incertidumbre educativa."

(ii) un enfoque descriptivo sobre la base de observaciones producidas en contextos de enseñanza en "ámbitos de enseñanza del proyecto" y (iii) un análisis de documentos sobre planificaciones y programas de los espacios curriculares que se ocupan de la formación en taller. ${ }^{2}$

\section{La prevalencia del "modelo tradicional" sobre la formación proyectual}

Si bien la formación del arquitecto es un proceso complejo, coordinado y holístico de saberes, destrezas, actitudes y competencias, es posible advertir ciertos espacios o áreas curriculares con una identidad propia: ${ }^{3}$ a) las ciencias básicas y tecnológicas, b) las ciencias sociales, planeamiento e historia, c) las morfologías y los sistemas de representación y d) las áreas de diseño, proyecto o "taller" de arquitectura. Centro mis análisis y afirmaciones sobre el conjunto de asignaturas que se ocupan de la formación de las aptitudes específicas del proyecto, considerando que poseen un "objeto de estudio" propio con dimensiones teórico-prácticas específicas sobre las cuales desarrollar sus contenidos, procesos de enseñanza y evaluación. La capacidad de proyectar implica un abordaje didáctico particular

2 Se han utilizado como insumos para el trabajo de campo los resultados de los trabajos de investigación colaborativa llevados a cabo en la asignatura Metodología de la Ciencia Aplicada al Diseño (FAU-UNNE), período 2014-2015, con mi dirección y coordinación general. Los programas y planificaciones corresponden a cátedras de taller de la carrera de Arquitectura de la UNNE y a otras universidades nacionales. Se analizaron, también, documentos vinculados con el proceso de acreditación de carreras de arquitectura.

3 En general, los diseños curriculares de las carreras de arquitectura en el ámbito nacional se estructuran sobre estas cuatro áreas académicas, que podemos definir como la integración de un conjunto de cursos, seminarios o talleres del plan de estudios que poseen elementos en común, seleccionados con criterio didáctico y pertinencia curricular, para abordar desde diversas perspectivas un ámbito común de prácticas, saberes y modos de generar nuevos conocimientos. Incluso esta idea se ha incorporado como un requerimiento en los estándares para la acreditación de las carreras, aprobada por el Consejo de Universidades e implementada por Coneau en los procesos de evaluación y acreditación. y una conceptualización de diseño coherente con su mecanismo de aprendizaje y formación.

Las estrategias pedagógicas — cómo se enseñadependen en estos espacios de la concepción que se tiene de la actividad — qué se enseña-. De allí se derivan un conjunto de preceptos y categorías teóricas que luego condicionan la manera en que se desarrolla la formación, la profesión y también la investigación. En el marco de las disciplinas proyectuales en general y en las asignaturas de proyecto en particular, se mantienen subyacentes dos supuestos o hipótesis de base, no siempre explícitas y, menos aún, críticamente adoptadas:

(i) la hipótesis del proyecto como un hacer-oficio, más que como una praxis-constitutiva y

(ii) la hipótesis de enseñanza del proyecto como una "simulación profesional" o una "práctica profesional supervisada".

Recupero aquí la revisión hecha por OXMAN (2001) sobre los paradigmas que persisten en la formación proyectual. En el marco de los estudios sobre designknowing and learning, OXMAN destaca tres modelos:

(i) el modelo del atelier o sistema de resolución de problemas simulando una situación de ejercicio profesional y guiado por un "experto";

(ii) el modelo de la formación a través del aprendizaje de los principios generales de la actividad introducidos en la ejercitación y;

(iii) el modelo de un marco cognitivo para el aprendizaje del proyecto (OXMAN, 2001: 271 yss.). Considero que es el tercer estadio de esta evolución lo que aún está pendiente de desarrollo y que las ideas de atelier-oficio y simulación profesional son todavía dominantes en la formación proyectual.

(i) El proyecto como un oficio. Desde este punto de vista, el proyecto arquitectónico se aprende como si fuera un oficio en el "atelier" del maestro. 
Las competencias esperadas son desarrolladas por el estudiante mientras realiza conductas motoras consistentes con la definición del nuevo objeto (arquitectónico) que se diseña, y alterna conversaciones reflexivas con su maestro-tutor y con los "materiales de la situación" proyectual. Los componentes de este modelo educativo fueron desarrollados por DONALD SCHON ${ }^{4}$ en 1983 y ampliamente difundidos por sus seguidores. Junto con la obra de HERBERT SIMON ${ }^{5}$, constituyen los aportes más relevantes y de mayor impacto en la investigación sobre el diseño y su enseñanza. Si bien es cierto que esta actividad conserva algo de su inspiración fundacional como un oficio ${ }^{6}$, en su proceso evolutivo ha ido adquiriendo complejidad, de acuerdo con los cambios en los contextos de influencia y de la propia dinámica de la creación arquitectónica. Actualmente, ya no es posible conceptualizar (y, consecuentemente, enseñar) el proyecto como un mero oficio, sino que es necesario considerar además otras categorías como la Profesión y la Disciplina7. El hecho de haberse instalado en las escuelas y facultades con estructuras formales y sistemáticas, con marcos normativos y legales que regulan su ejercicio, es una muestra de que el proceso de institucionalización se basa en la construcción de un conocimiento especializado

4 Estas ideas germinales de SCHÖN tienen su origen en los trabajos realizados en conjunto con CHRIS ARGYRIS, en la búsqueda de las dinámicas que conducen al aprendizaje reflexivo y en los procesos de formación de diferentes tipologías de profesionales.

5 Por ejemplo, la definición de SIMON que considera el diseño como una forma de transformar situaciones existentes en otras más convenientes es la más citada de la bibliografía en diferentes ramas de aplicación del diseño.

6 No se intenta aquí descartar la importancia que tiene el manejo del "hacer" proyectual, dado que el aprendizaje del diseño requiere necesariamente el desarrollo de competencias prácticas, sino solamente marcar la insuficiencia de este criterio que debe ser reconsiderado a la luz de los avances en la disciplina y sus procesos de producción.

7Disciplina no en el sentido clásico (teórico), sino como trans-disciplina o proceso de transducción de diferentes aportes en la constitución de nuevos artefactos con una base cognitiva singular. y en la consolidación de competencias específicas que permiten un control sobre la actividad. La profesión va más allá del mero oficio, que no se rige por estudios formales y que replica rutinariamente las características de una ocupación habitual.

Considero importante pensar en las diferencias de abordaje y comprensión que se producen al pasar de una concepción del proyecto como "oficio" a otra que intenta visualizar su estructura y dinámica desde el punto de vista de una Praxis Disciplinar. Una disciplina es fundamentalmente un campo de conocimientos en evolución y transformación. Implica una compleja y explícita articulación entre un conjunto de prácticas y de conocimientos involucrados en la tarea, además de los procesos sistemáticos de reflexión sobre ambas faces o instancias proyectuales. Cuando - como en el caso de la Arquitectura - el objeto de estudio pasa (ultrapasa) a través de diferentes disciplinas, se lo considera una trans-disciplina ${ }^{8}$. Aceptar este enfoque implica también reconocer nuevas funciones del proceso proyectual, dimensiones cognitivas concretas - y su análisis explícito- que no forman parte habitual de la formación en diseño.

(ii) La hipótesis de la simulacion profesional. La enseñanza del proyecto arquitectónico está basada — de manera preponderante - en la replicación del desempeño de actividades consistentes con el ejercicio profesional. Los indicadores del desempeño educativo-formativo del alumno son análogos a los que regulan la producción de un

8 La transdisciplinariedad fue definida por BASARAB NICOLESCU mediante tres postulados metodológicos: la existencia de niveles de realidad, la lógica de los intermedios incluidos y la complejidad. En presencia de variados niveles de realidad, el espacio entre las disciplinas y más allá de las disciplinas está lleno de información. Las investigaciones en torno a cada disciplina implican, como mucho, un mismo nivel de realidad, aunque, en la mayoría de los casos, solo quedan involucrados algunos fragmentos de un cierto nivel de realidad. Por el contrario, la transdisciplinariedad implica la dinámica engendrada por la acción de varios niveles de la realidad a un mismo tiempo, tal como se manifiesta en el proyecto. 
"Es un imperativo categórico que tanto las dimensiones prácticas como las teóricas (acciones y conocimientos) deben quedar explicitados en el proceso pedagógico, conceptualizando así al diseño como una praxis en lugar de un mero hacer efectivo."

objeto de diseño. Su aplicación está basada en el desarrollo de habilidades (tales como en el oficio) para la conducción de una simulación de un proceso real.

Este modelo conserva el fundamento del talleratelier o estudio de arquitectura y de la enseñanza tradicional, a través de la práctica supervisada de la solución arquitectónica de un proyecto. La supervisión se alimenta de correcciones sucesivas que orientan la evolución exitosa del proceso.

El diseño, entonces, se representa como una simulación de la práctica profesional y la demostración es el argumento principal de su enseñanza. El aprender haciendo (learning-by-doing) y el modelo del espacio o desarrollo proximal ${ }^{9}$ son discursos habituales para justificar las reglas de la "transmisión" más o menos tácita de los conocimientos que están en juego en cada aprendizaje. Si bien es cierto que toda actividad que se realiza de manera eficaz implica necesariamente un conjunto de conocimientos específicos que se "ponen-en-acto" en el momento de realizar una acción, se corre el riesgo de que estos conocimientos queden "ocultos", implícitos e inaccesibles detrás del aprendizaje de las rutinas proyectuales. Es algo así como un saber hacer (Know How), pero disociado del saber qué (Know What) y del saber por qué (Know Why). No desconozco el valor del conocimiento tácito (POLANYI, 2009) y su aplicación a múltiples actividades humanas, aunque considero preciso convertirlo en un tema de trabajo y reflexión para sacar un provecho educativo de su uso. Algo similar a lo propuesto por NONAKA Y TAKEUCHI (1995) para convertir en explícito el conocimiento tácito de una organización que se interesa por gestionar el conocimiento como argumento de calidad.

9 Este modelo fue desarrollado por VIGOTSKI a principios del S. XX describiendo los procesos de aprendizaje que se producen de manera implícita en la relación maestro-discípulo. La zona de desarrollo proximal (ZDP) refiere a la brecha entre las habilidades de un alumno y las que puede adquirir con la guía de un maestro o tutor.
Es un imperativo categórico que tanto las dimensiones prácticas como las teóricas (acciones y conocimientos) deben quedar explicitados en el proceso pedagógico, conceptualizando así al diseño como una praxis en lugar de un mero hacer efectivo. El concepto de praxis se focaliza necesariamente en el estatus epistemológico del proyecto, en sus fundamentos y funciones cognitivas básicas.

Así, lo que se enseña y aprende comprende al mismo tiempo tanto procedimientos u operaciones gráfico-modelizantes, como conocimientos y procesos lógicos de pensamiento propios de la actividad $^{10}$

Surgen de este contexto las preguntas conexas del presente artículo: ¿cómo puede el conocimiento proyectual ser adquirido o aprendido?; ¿es el proyecto solamente un oficio?; ¿cómo podemos redefinir la tarea del aprendizaje en diseño, pasando de una visión basada — casi exclusivamente- en la producción de un objeto (artefacto) a una visión que integre además a las dimensiones cognitivas del pensamiento proyectual?

Como vimos, la respuesta clásica a la formación proyectual es que se pone en práctica un "aprender haciendo" (learningbydoing), pero ¿qué es lo que se aprende haciendo? ¿Cuál es el objeto de aprendizaje que permite desarrollar las habilidades típicas (específicas) de todo diseñador? En este sentido, se confunde con frecuencia el tema de proyecto (vivienda, escuela, centro deportivo, etc.) con el objeto de conocimiento que la práctica sobre estos problemas genera. ¿Dónde se ubica, entonces, este "objeto" central de conocimiento: en el docente, en el alumno, en las prácticas, en los resultados...? Es claro que las concepciones dominantes del proyecto

10 NIGEL CROSS (1984, 2007) acuñó en los 80 —y luego lo desarrolló más - el concepto de "designerly ways of knowing" para referirse a una manera proyectual (o propia del proyecto) de construir o desarrollar conocimiento, diferente del de la ciencia o la tecnología. 
como un "oficio" y un programa metodológico dominan el escenario dejando dudas sobre el modo en que efectivamente se construyen los nuevos conocimientos para avanzar hacia niveles de experticia más complejos como camino de formación de cada proyectista hacia niveles de competencia deseados (DORST, 2008).

\section{Elucidando el concepto de "conocimiento proyectual"}

Conel supuesto denuevas dimensionesconstitutivas de la praxis proyectual es preciso, en este contexto, replantear el modelo de abordaje para hacer visible el conocimiento que se genera en el proceso de diseño, que en palabras de OXMAN se produce en un proceso creativo constructivo, donde " $t]$ he constructional form provides a representation of the structure of knowledge, whichth estudents acquire through constructing. Learning may be considered a process of knowledge acquisition and development in which the knowledge, or a representation of the knowledge, is physically constructed" (2001: 281).

Considerar el aprendizaje como un proceso de adquisición o construcción de conocimiento proyectual requiere hacer explícitos los procesos involucrados en su producción: ¿cómo construye el alumno nuevas estructuras de aprendizaje, conocimiento y razonamiento proyectual? Aun cuando sea actualmente un tema de debate, es evidente que el proyecto genera conocimiento. Este conocimiento posee un estatus epistemológicorepresentacional, derivado de los procesos de modelización e investigación, la formulación de problemas, el uso de lógicas generativas y evaluativas y la construcción de representaciones.

La dimensión cognitiva tiene que ver precisamente con el contexto simbólico en el que se vehiculizan las ideas de diseño, a través de una amplia variedad de herramientas propias del proyecto. La índole de este conocimiento es relevante para elucidar conceptual y operacionalmente el problema genuino ${ }^{11}$ que encierra todo proyecto, exponer su estructura y dinámica interna, orientando de esta manera los procesos de re-solución y respuesta. Pareciera, entonces, que la cuestión central gira en torno del conocimiento que el diseñador (desde niveles nóveles hasta avanzados) va construyendo en su formación y perfeccionamiento. Este conocimiento proyectual es la base de la competencia de todo arquitecto, es el núcleo central de su capacidad proyectante. SILVA (2002) sugirió la necesidad de conocer el "aparato cognitivo esencial" del arquitecto para así coordinar los procesos de formación y las posibles equivalencias del título entre diferentes países del Mercosur.

Ahora bien, ¿qué contiene ese aparato cognitivo esencial?, ¿cómo se lo construye?, sobre estas cuestiones no existen mayores precisiones, aunque se supone que es lo que logra capitalizar el estudiante en su carrera y le permite resolver cualquier problema-situación de proyecto, haya formado parte como tema de diseño de su formación o no. Salta a la vista que aquello que habitualmente se concibe que se-aprende-haciendo excede el ámbito del tema-problema que se está resolviendo y se generaliza, como una competencia, hacia potenciales situaciones de acción proyectual. En concreto, lo que se aprende diseñando una vivienda queda como patrimonio del diseñador para cualquier otra situación de diseño, sea otra vivienda, un espacio público o un edificio educativo. El conocimiento proyectante se construye en una experiencia particular, pero es llevado a una categoría general para su aplicación en diferentes contextos. La dimensión cognitiva del diseño apunta

11 Un problema genuino existe (en el campo proyectual) cuando a partir de una particular condición de necesidad, demanda o carencia, la solución no es evidente y hace necesario conducir (de manera consciente) un proceso de modelización, investigación, producción y evaluación como acción eficaz fundada en el conocimiento. 
"Considerar el aprendizaje como un proceso de adquisición o construcción de conocimiento proyectual requiere hacer explícitos los procesos involucrados en su producción."

a un conocimiento implicado en la acción proyectual para poder producir una respuesta coherente y efectiva a cualquier situación problemática derivada de una experiencia de intervención concreta.

Pero este conocimiento no es del tipo informativo, no es un dato tal como ¿cuál es la capital de Argentina? o ¿cuál es la tensión admisible del acero? No es un conocimiento que puede ser "transmitible" o "enseñable" con los procedimientos tradicionales. Tampoco implica una experiencia o una experticia, como saber dibujar o saber dónde se colocan los puntos de fuga o cómo se logra una vista aérea en un croquis. Es un conocimiento más complejo, y es esta misma complejidad y obscuridad que lo rodea lo que impacta en las estrategias didácticas utilizadas, que no logran apuntalar consistente y explícitamente a su producción.

GOEL ha aportado un dato interesante: el conocimiento del diseñador está disociado en dos tipos: 1) un conocimiento específico referido a un dominio particular y 2) un conocimiento procesual o procedimental. En términos de GOEL, los diseñadores requieren dos tipos de conocimiento: "explicit articúlate, domain-specific knowledge, and inarticulate, domain-independent, procedural knowledge" (2001: 221). Esta es una distinción funcional y no meramente especulativa. Por mi parte he logrado detectar en el análisis de los datos que los conocimientos tipo 1 son aquellos que podemos adquirir a través del estudio, de la transmisión o de la atención a experiencias de otros; son saberes ya sistematizados y organizados disciplinarmente. Sin embargo, los conocimientos del tipo 2 son típicamente operatorio-metodológicos y exponen el modo en que se toman las decisiones acudiendo a un conocimiento implicado en el proceso y en una situación particular ${ }^{12}$. Los primeros son explícitos o "de tenencia" y los segundos son implícitos o "de

12 En otro lugar he equiparado el diseñar con el investigar-proyectualmente, siendo esta acción proyectante un similar a un design by research o diseñar a través de la investigación (BURGOS, 2015). construcción". Este conocimiento implícito que se construye en el proceso no parece subordinarse a nuestro concepto clásico de conocimiento; sin embargo, lo es.

Aunque esta dificultad es la que le confiere cierta obscuridad, invisibilidad o indeterminación en el momento de convertirlo en el eje de una estrategia formativa. Es el conocimiento que da estructura y dinámica al método de diseño, aunque visto desde un punto de vista descentrado de las clásicas concepciones que consideran al método como un programa rígido o un conjunto de pasos. El método siempre funciona como un "ensayo", una conjetura, un "camino estratégico". MORIN (2003) ha hecho una excelente contribución para alejar la idea de método de la concepción clásica de la "receta". Desde su punto de vista, el método es "obra de un ser inteligente que ensaya estrategias para responder a las incertidumbres. En este sentido, reducir un método a un programa es creer que existe una forma a priori de eliminar la incertidumbre" (2003: 32).

Quizás la función más importante del método sea la del aprendizaje y no la de pre-establecer el proceso; este aspecto expresa la diferencia entre la función prescriptiva (rígida) y la función cognitiva (constructiva) de los métodos. En este sentido, se entiende método como un "reaprender a aprender en un caminar sin meta definida de antemano. Reaprender a aprender con la plena conciencia de que todo conocimiento lleva en sí mismo y de forma ineliminable la marca de la incertidumbre" (MORIN, 2003: 68-69). No olvidemos que para el estado actual de conocimiento sobre los problemas que aborda el proyecto, la indeterminación y la incertidumbre son las constantes o la norma y no la excepción en el proceso, y donde los problemas han sido concebidos como inciertos, indeterminados o mal estructurados. Esta conclusión es contundente y no es discutida en la actualidad, desde que COYNE lo ha expresado con 
total contundencia afirmando que "[w]ickednessis the norm. It is tame formulations of professional analysis that stand out as a deviation" (2005: 12).

Entonces, tenemos un conocimiento transferible, explícito, comunicable, que puede ser gestionado por medios educativos más o menos tradicionales y otro no-transferible, implícito y no comunicable, que requiere una construcción activa — reflexivapor parte del diseñador y no puede ser abordado desde lógicas didácticas típicas, como la simulación profesional o la práctica supervisada. Podemos, incluso, distinguir entre un conocimiento ya formado y establecido y un conocimiento en formación que surge como resultado de la acción metodológica y cognitiva del método y, con ello, una epistemología conceptual o teórica y una epistemología operatoria o praxeológica ${ }^{13}$.

El conocimiento proyectual se construye en situaciones no rutinarias, complejas y cargadas de cierto contenido de incertidumbre, como todas las de diseño genuino. Es lo que confiere al proyecto su contenido innovador o creativo, no es una respuesta repetitiva o reproductiva, sino esencialmente original (anclada en parámetros, demandas y condicionantes particulares de la situación de intervención). Autores ya clásicos, COMO SIMON (1973), RITTEL Y WEBBER (1973), BUCHANAN (1992), COYNE (2005) y FARRELL (2013), han definido el problema o la situación de diseño como ill-structured; ill-definied o wickedproblems and situations, aludiendo a la dificultad que presenta para ser configurada y determinada. Sin embargo, esta situación de complejidad e indeterminación es lo que posibilita que el conocimiento proyectual pueda ser construido, ya que es condición fundamental para que el diseñador defina mecanismos computacionales,

13 Podríamos poner aquí múltiples ejemplos de este tipo de saberes implicados en prácticas o acciones. Por ejemplo, existe consenso en afirmar que a investigar se aprende investigando y - mutatis mutandi- a diseña se aprende diseñando. La construcción del conocimiento es el resultado de una práctica reflexivo-cognitiva con centro en el sujeto que la realiza. No es correcto decir que se enseña a otro a investigar, diseñar, andar en bicicleta: jes el protagonista mismo quien lo aprende! conceptuales, operacionales y representacionales para abordar-resolver el problema. Estos conocimientos requieren los procesos de la práctica, del ensayo, de la experimentación y la exploración reflexiva y crítica. Solo se exponen en el "ir a tientas" de todo proceso genuino de proyecto. Son conocimientos implicadosen-la-acción y son visibles únicamente en-la-acción proyectual, a-través-de la acción o a-partir-de la acción proyectual por algunos procesos cognitivos que actúan como "líquido revelador" de su configuración y de su dinámica.

Es evidente que el conocimiento que trato aquí actúa como una cupla entre el manejo de saberes (conocimientos en sentido clásico) y operaciones o procedimientos, con el fin de conducir un proceso de modelización complejo. Es desde el punto de vista que presento aquí el método. Es el método, entonces, el conocimiento que guía una cierta praxis constitutiva y re-constructiva que realiza el diseñador. Una praxis modelizante, re-constructiva y experimental: una praxis proyectante. Por ello propongo equiparar este conocimiento implicado en la acción con una idea - no trivial- de método y de praxis proyectante estratégica.

Es, entonces, la praxis una acción protagónica generativa que busca, experimenta y orienta estratégicamente el proceso hacia la toma de decisiones consistentes con la situación de intervención, compleja, incierta, multidimensional. Lo interesante de esta acción es que no solo produce un resultado para concebir el artefacto arquitectónico, sino que en el mismo movimiento de su manifestación se conoce a sí misma, aprende en el mismo camino y emerge en la misma experiencia que genera. Por ello he afirmado ya en otro lugar (BURGOS, 2014) que es necesario desplazar la atención desde la función prescriptiva de los métodos hacia una función cognitiva en la que se produce un aprendizaje reflexivo y estratégico de la actividad. Una de las consecuencias de esta afirmación es que no podemos ya sostener la 
"El conocimiento proyectual se construye en situaciones no rutinarias, complejas y cargadas de cierto contenido de incertidumbre, como todas las de diseño genuino."

visión del método como una receta, un programa con un sesgo determinista y con la expectativa de un orden preestablecido de pasos, fases o etapas, sino como un proceso reflexivo-praxeológico.

La dinámica del eje cognitivo-metodológico en la praxis proyectante permite articular las funciones del pensamiento implicado en el proyecto con el modelo metodológico que sostengo aquí. Incluso, es posible afirmar que son las funciones del pensamiento proyectual las que alimentan la dimensión cognitiva del método. El pensamiento de un diseñador pone en acto redes de funciones complejas, como la representación, la modelización, la simbolización, la investigación. Esta última como estrategia de determinación, exploración y síntesis de lo diverso y lo incierto (DEWEY, 1938). La dimensión reflexiva popularizada por SCHÖN permite realizar el giro hacia los propios procesos de la práctica, posibilitando la generación de nuevas propuestas a partir de la validación y adecuación de sus atributos. Por otra parte, la imaginación creadora y el razonamiento crítico parecen ser dos dimensiones de un mismo proceso cognitivo, al tener que aportar nuevas ideas y alternativas a cuestiones concretas de la demanda, el contexto y los condicionantes propios de una situación real de intervención. Esta acción metodológica en un registro cognitivo emerge como resultado del uso de símbolos y la creación de un lenguaje, la gestión de diferentes procesos operacionales, el aprendizaje situado y contextualizado, el uso de información relevante, la realización de razonamientos y mecanismos argumentativos, que posibilitan la toma de decisiones con fundamento, la resolución de problemas derivados del análisis de contextos reales, etc.

\section{Modelo de la estructura de la praxis y el rol del conocimiento proyectual}

Considerando, entonces, la propuesta inicial de GOEL podemos llevarla un poco más allá y enriquecer su presentación. Sabemos que hay un conocimiento ya formado, disponible y explícito que alimenta la cultura arquitectónica del diseñador. Como derivado de este saber explícito voy a proponer la existencia de un conocimiento situacional propio del contexto particular de la situación de proyecto. Ambos conocimientos configuran un eje estructurante y explícito de conocimiento disciplinar. En una dirección o eje dinámico ubicamos, entonces, el conocimiento que GOEL ha denominado procedimental o procesual y que he denominado método, junto al pensamiento proyectual que - como he sugerido- da a la acción de proyecto un carácter propio y una identidad disciplinar.

Toda situación particular de intervención demanda conocimientos específicos vinculados con el tema, las demandas y las condicionantes particulares. Este saber contextualizado se suma al saber disciplinar que todo diseñador maneja y que es específico de su práctica: el manejo de las funciones, las necesidades, los tratamientos formales y espaciales, las respuestas tecnológicas, etc. En este eje se configuran los conocimientos explícitos de la praxis. Todo allí es comunicable y puede ser considerado desde una epistemología conceptual o teórica.

Por otra parte, se juegan otros procedimientos igualmente importantes que configuran el eje dinámico del proceso y conjugan el pensamiento proyectual y lo que considero el conocimiento proyectual esencial, que es el método. El pensamiento humano, en el marco del proyecto, tiene una identidad particular, por los objetivos que persigue, los recursos que utiliza y las condiciones internas-externas sobre las cuales trabaja. Su función básica es modelizar ideas, conceptos, situaciones y disposiciones tecnológicas para traducir las condiciones del sitio, la demanda y el contexto a las cualidades de un objeto arquitectónico destinado al hábitat humano. En este proceso de modelización 
se re-construyen de modo original significados y "hechos" en una dinámica que lleva consigo el sello de la experimentación y la exploración (investigación) permanente, de modo lógico, crítico y reflexivo. El método (más allá de las concepciones triviales e ingenuas de las secuencias) tiene la doble condición de generar propuestas y ponerlas a prueba para justificar los resultados. Requiere, por lo tanto, la construcción de un marco lógico de referencia donde se localicen los aspectos que permitirán realizar una evaluación o contraste permanente sobre la calidad de cada idea o propuesta y un sistema de representaciones que pueda captar la complejidad del fenómeno. Estas representaciones exceden el ámbito de las tipologías especulares o isomórficas (plantas, visualizaciones, maquetas usadas para representar lo que ya existe) y se extienden hacia la búsqueda, capturas o especificaciones de nuevas formas o ideas que aún no existen.

En un esquema presento gráficamente la primera versión (sintética) del modelo:

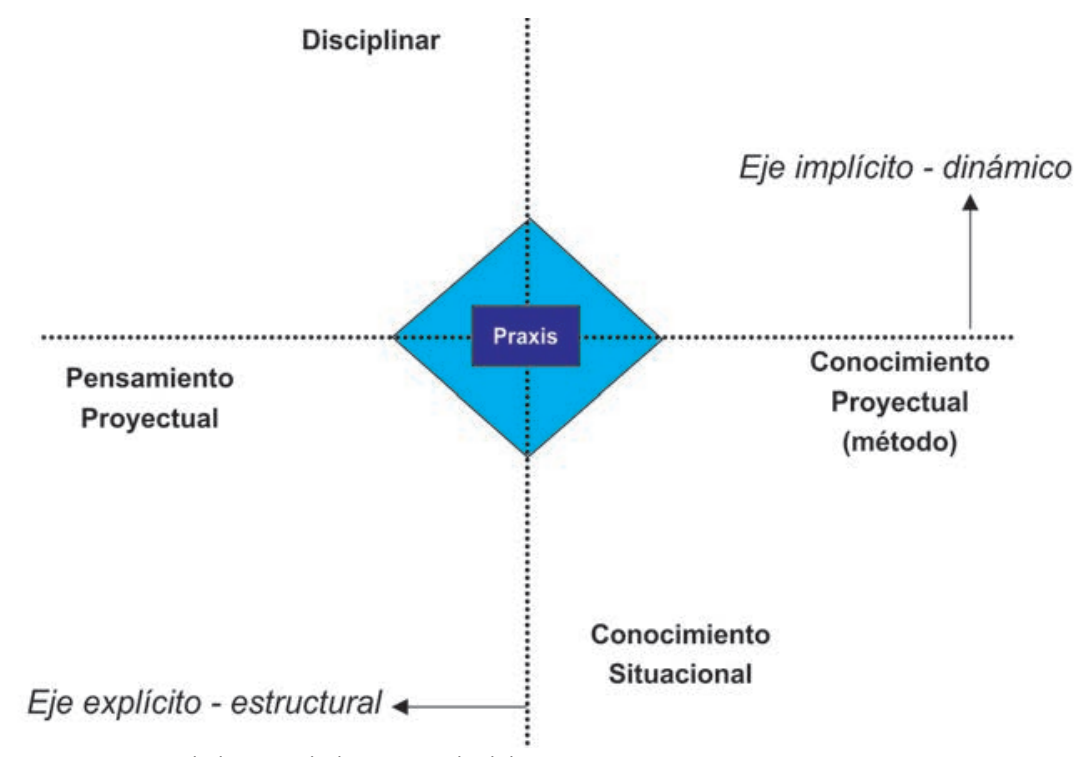

Gráfico 1. Componentes cognitivos de la actividad proyectual. Elaboración propia

Se advierte en el gráfico 1 que la praxis es el resultado de la articulación de diferentes tipos de conocimiento implicados en la acción. Sobre el eje explícito o estructural se opera educativamente con buenos resultados, bajo estrategias convencionales. Sin embargo, el eje implícito o dinámico presenta múltiples obstáculos, dado que sus contenidos exponen un alto grado de complejidad e indefinición.
El modelo posee, entonces, cuatro variables principales: (i) el conocimiento disciplinar, (ii) el conocimiento situacional, (iii) el pensamiento proyectual y (iv) el conocimiento proyectual o método. A su vez, cada una de ellas posee dimensiones $\mathrm{O}$ atributos particulares. El conocimiento disciplinar se constituye con las configuraciones funcionales, espaciales, formales y tecnológicas. El conocimiento situacional se 


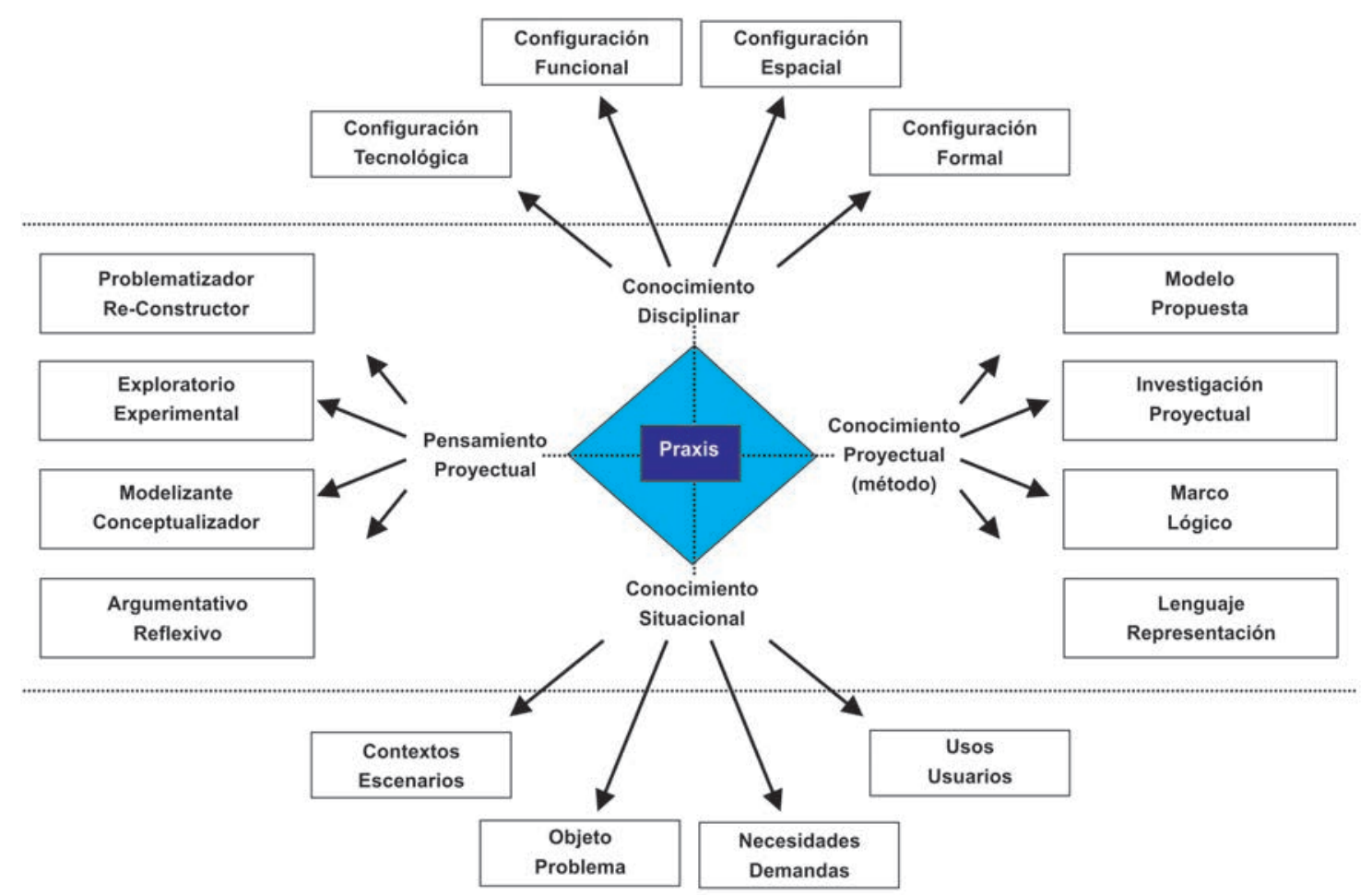

Gráfico 2. Modelo de diferentes tipos de conocimiento implicados en la praxis proyectante. Elaboración propia

expresa a través de los contextos-escenarios, las necesidades-demandas, el objeto-problema y los usos-usuarios. El pensamiento proyectual posee como atributos o identidades principales ser problematizador-reconstructor, exploratorioexperimental, modelizante-conceptualizador y argumentativo-reflexivo.

En un nuevo esquema podemos mostrar, con mayor detalle, los componentes de la praxis proyectante con sus diferentes tipos de conocimiento implicados en la acción, desde dos ejes: uno estructurante o explícito (vertical) y otro dinámico o metodológico (horizontal).

\section{La crítica educativa que el modelo deja planteada}

El eje explícito (vertical), desde una epistemología conceptual o "de tenencia" con la cual se fundamentan los conocimientos estructurantes de la actividad de proyecto y pueden ser comunicados por medios tradicionales: la transmisión de experiencias, las clases, los textos y producciones sistematizadas, etc. Sin embargo, el eje implícito (horizontal), regido por una epistemología de la praxis, la experiencia protagónica y la acción estratégica orientada a fines, presenta obstáculos pedagógicos importantes tanto para los diseños curriculares como para la definición de "objetos de estudio" vinculados con la dimensión cognitiva del método. Es muy poco frecuente encontrar actividades orientadas a trabajar los procesos de pensamiento proyectual, la investigación proyectual, las instancias de modelización, los diferentes tipos representacionales (isomórficos y homológicos), las formas clásicas de argumentación y construcción de sentido, la construcción de marcos lógicos de referencia y contexto, etc. Por ello todos estos conocimientos permanecen en la penumbra de la actividad del diseñador. No sabemos en qué grado se desarrollan, por qué o cuándo se activan y aplican. Se da por hecho que el estudiante sabe reflexionar (incluso en el sentido de SCHÖN), modelizar, argumentar, conceptualizar, comprender e interpretar, evaluar y decidir, investigar y experimentar proyectualmente, aunque estas funciones no son parte de la propuesta educativa de nuestras carreras o, si lo son, se presentan de modo ciertamente marginal.

Por ello, considero importante diseñar, programar e implementar - junto con las modalidades de enseñanza centradas en la simulación y en la práctica profesional supervisada- otras estrategias orientadas a desarrollar habilidades y competencias en el manejo de dimensiones típicas del pensamiento proyectual y del método como praxis cognitiva.

El método, a partir del giro propuesto en este artículo, ya no se considera un programa explícito de secuencias jerárquicas o de pasos. Esto implicaría abandonar - $\mathrm{O}$ al menos matizarlas funciones prescriptivas y avanzar hacia las funciones cognitivas, es decir, trabajar sobre el aprendizaje de las invariantes del método como camino estratégico que se piensa en movimiento 
para afrontar la incertidumbre, la complejidad de los escenarios, la toma de decisiones fundadas y promover la autodeterminación del diseñador y el control de sus propios procesos productivos. Desde el punto de vista didáctico implica prever estrategias que focalicen el proceso de diseño como tema-objeto de aprendizaje, aunque no como un saber previo, sino desde una actitud reflexivo-crítica para hacer conscientes los procesos de respuesta y toma de decisiones que intervienen en un proyecto y focalizarlos como tema de estudio. Podríamos tomar por casos (i) la construcción de una situación problemática de intervención, (ii) la formulación de un problema de diseño como sistema jerárquico de variables y relaciones, (iii) la producción de alternativas innovadoras en la resolución y respuesta, (iv) la creación de modelos que interpretan (reconstruyen) las demandas y necesidades de los usuarios, (v) la creación de nuevas categorías conceptuales que encarnan simbólicamente en los objetos arquitectónicos transportando nuevas entidades de sentido hacia sus escenarios de uso y apropiación, entre muchos otros.

\section{CONCLUSIONES}

Conforme con el objetivo principal de este trabajo he aportado un modelo de la praxis proyectante basado en un conjunto de diferentes tipos de conocimiento articulados en función de saberes ya disponibles y otros que se construyen en el mismo proceso de diseño. En este sentido, he ubicado al método en un registro diferente de la visión clásica colocándolo en un eje dinámico-constitutivo como pensamiento proyectual particular. En este sentido he sugerido algunas críticas al modelo educativo tradicional y su inadecuación para gestionar objetos de estudio complejos y procesuales y esbozado algunas ideas para incorporar nuevas estrategias educativas en las disciplinas proyectuales de la formación del arquitecto.

\section{REFERENCIAS BIBLIOGRÁFICAS}

BUCHANAN, Richard (1992). "Wicked Problems in Design Thinking". Design Issues, vol. 8, no. 2, pp. 5-21.

BURGOS, Carlos (2014). La praxis constructiva del diseño tecnológico: un análisis de sus dimensiones ontológicas, epistemológicas y metodológicas. [Disertación de doctorado] Donostia-San Sebastián, Universidad del País Vasco/Euskal Herriko Unibertsitatea, 428 pp.

-------(2015). " Más allá del modelo del problem-solving: el proyecto arquitectónico como investigación proyectual". ARQUISUR, Revista N. ${ }^{\circ} 7$, pp. 20-32. ISSN - 1853-2365.

COYNE, Richard (2005). "Wicked Problems Revisited". Design Studies, vol. 26, no. 1, pp. 5-17.

CROSS, Nigel (1984). Developments in design methodology. John Wiley \& Songs: New York

-(2007).Designerly Ways of Knowing. Birkhäuser Verlag: Berlin. DEWEY, John (1938).The Theory of Inquir., Henry Holt and Company: New York.

DORST, Kees (2008). "Design Research: A Revolution-Waiting-toHappen". Design Studies, vol. 29, no. 1, pp. 4-11.

GOEL, Vinod (2001). Dissociation of design knowledge. En: C. Eastman M. McCracken \& W. Newstetter (eds.), Design Knowing and Learning: Cognition in Design Education, (pp. 221-269), Elsevier Science: Oxford. FARRELL, Robert, HOOKER, Cliff (2013). "Design, Science and Wicked Problems". Design Studies, vol. 34, no. 6, pp. 681-705.

MORIN, Edgar, CIURANA, Emilio, MOTTA, Raúl (2003). Educar en la era planetaria: el pensamiento complejo como método de aprendizaje en el error y la incertidumbre humana. Barcelona: Gedisa.

NONAKA, Ikugiro, TAKEUCHI, Hirotaka (1995) The knowledgeCreating Company: How Japanese Companies Create the Dynamics of Innovation. Oxford University Press: New York.

OXMAN, Rivka (2001). The Mind in Design: A Conceptual Framework for Cognition in Design Education. En: C. Eastman, M. McCracken \& W. Newstetter (eds.), Design Knowing and Learning: Cognition in Design Education, (pp. 269-295), Elsevier Science: Oxford.

POLANYI, Michael (2009) The Tacit Dimension. University of Chicago Press: Chicago.

SCHON, Donald (1983) The Reflective Practitioner: How Professionals Think in Action. Basic Books: New York.

SILVA, Elvan (2002). "El Aparato cognitivo esencial en la formación de arquitecto". Documento de integración curricular - CODFAUN. Inédito. SIMON, Herbert (1973). "The Structure of III Structured Problems". Artificial Intelligence, vol. 4, pp. 181201.

RITTEL, Horst, WEBBER, Melvin (1984). Planning Problems are Wicked Problems. En: N. Cross, (ed.), 1984, pp. 135-144. 